\title{
PERCEPCIÓN SOBRE LA IGUALDAD EN JÓVENES ADOLESCENTES Y EL ROL DE LA EDUCACIÓN
}

\author{
María del Mar González López \\ Grupo de Investigación Humanidades - 498 \\ Universidad de Almería \\ Remedios López Liria \\ Profesora Departamento Enfermería, Fisioterapia y Medicina \\ Universidad de Almería \\ rll040@ual.es
}

Recepción Artículo: 14 diciembre 2020

Admisión Evaluación: 14 diciembre 2020

Informe Evaluador 1: 15 diciembre 2020

Informe Evaluador 2: 15 diciembre 2020

Aprobación Publicación: 16 diciembre 2020

\section{RESUMEN}

El presente trabajo trata de abordar un tema actual y de carácter social, la igualdad entre hombres y mujeres. Históricamente, se ha llevado a cabo una lucha imparable por parte de las mujeres para alcanzar la plena igualdad de derechos y libertades con respecto a los hombres. Aún en la actualidad, existen diferencias que perjudican a éstas y que se debe continuar tratando de erradicar. La educación desarrolla un papel crucial en la adquisición de estos valores, pues si el cambio es posible, está en mano de las futuras generaciones.

El objetivo de este estudio ha sido el análisis de la percepción del alumnado de secundaria con respecto al tema. Metodología: se obtuvieron los datos de 182 participantes a través de una encuesta online durante la primavera de 2020. Resultados: En lo referente a los estereotipos ligados al genero, se destaca a nivel general un concepto positivo sobre los mismos. Sin embargo, al tratar el tema del amor, los porcentajes varian a favor de la toxicidad y perdida de la privacidad personal. Lo mismo sucede con respecto al trabajo, donde la mitad de los participantes no consideran que exista discriminacion en este ambito.

Conclusiones: Según la literatura revisada y las respuestas de los jóvenes, se denota la necesidad de una educación que trate y abogue por la no discriminación por razón de género.

Palabras clave: igualdad; educación; valores; feminismo; cambio; coeducación

\section{ABSTRACT}

Perception of equality in young adolescents and the role of education. The present project aims to treat one of the most current topics although it is not something new: equality between men and women. Historically, there has been an unstoppable struggle by women to achieve full equality of rights and freedoms with men. Even today, there are differences that are detrimental to them and 


\section{PERCEPCIÓN SOBRE LA IGUALDAD EN JÓVENES ADOLESCENTES Y EL ROL DE LA EDUCACIÓN}

that must continue to be eradicated. Education plays a crucial role in the acquisition of these values, because if change is possible, it is in the hands of future generations.

The aim of this study is to analyse the perception of secondary school students with regard to this subject. Methodology: data from 182 participants were obtained through an online survey during the spring of 2020. Results: Regarding gender stereotypes, a positive concept about gender stereotypes is highlighted at a general level. However, when dealing with the topic related to love, the percentages vary in favour of toxicity and loss of personal privacy. The same applies to work, where half of the participants do not consider that there exists discrimination in this area.

Conclusions: According to revised literature and participants' responses, there is a need for education that addresses and advocates gender non-discrimination.

Keywords: equality; education; values; feminism; change; coeducation

\section{ÁREA TEMÁTICA Y ANTECEDENTES}

Vivimos en una sociedad constituida por diversos valores sociales que nos caracterizan como seres humanos, entre los que destacan la tolerancia, la justicia, el amor, el respeto, la paz, la honestidad y la igualdad, entre muchos otros. Con el presente trabajo, se pretende abordar el tema de la igualdad desde una perspectiva de género y en la educación. Se analiza si existe una situación discriminatoria de la mujer desde la perspectiva de los jóvenes.

La escuela es el lugar donde se aprende sobre numerosas materias y ramas relacionadas con las letras y las ciencias, pero además donde se ponen de manifiesto los valores que hacen de los individuos unas personas tolerantes. Además, no todo el alumnado dispone del mismo contexto socio-económico y familiar, lo que marca en mayor o menor medida el acceso a una educación de calidad en casa.

La educación es la base para formar a personas responsables y respetuosas con los demás, y la mejor herramienta para formar en pensamientos no discriminatorios por razones de sexo. Así se produce el nacimiento de la llamada coeducación, definida por Martori (2009) como: "El termino «coeducacion» es utilizado comunmente para referirse a la educacion conjunta de dos o mas grupos de poblacion netamente distintos: aun cuando ha sido a veces aplicado a la educacion conjunta de grupos formados sobre la base de caracteristicas de diversos tipos -clase social, etnia, etc.-, su uso habitual hace referencia a la educacion conjunta de dos colectivos humanos especificos: Ios hombres y las mujeres." (p. 94)

Por su lado, Extremera y Montero consideran la coeducación como un proceso de socialización: "Un proceso de socializacion humana centrado en las alumnas y alumnos, considerados como grupos con igualdad de derechos y oportunidades. Se basa en la ideologia de la igualdad, a la que anade la libertad, la diferencia y la solidaridad" (2009, p.111).

Otra aportación significativa es la de Raedo (2014), que añade que además es preciso que los docentes tengan en cuenta estas diferencias con la finalidad de erradicarlas en todos los ámbitos posibles. Por ello, es fundamental aportar al alumnado espacios libres de estereotipos y discriminaciones por cuestiones de género (Colás y Villaciervos, 2007).

La coeducación ha de estar presente en todos los espacios de una escuela, desde los valores de los docentes, como en el contenido de las asignaturas y por supuesto, en la detección de comportamientos discriminatorios por parte del alumnado. De esta forma, tanto niñas como niños comparten un entorno y pueden ser capaces de desarrollar su propia identidad sin crear ningún prejuicio, sin un lenguaje discriminatorio, por ejemplo (Raedo, 2014). De esta forma, a largo plazo se puede lograr que "generaciones futuras crezcan libres de estereotipos y tengan relaciones más igualitarias. Liberará a las mujeres de la opresión machista y a los hombres de asumir otros estereotipos y papeles que no deben aceptar jamás. Toda la sociedad gana” (Bernal, 2019, p.7). 


\section{OBJETIVOS}

-Conocer el grado de percepción de los jóvenes frente a la diferencia social entre hombres y mujeres.

-Valorar la necesidad e importancia de una educación que abogue por la igualdad de género de forma directa entre el alumnado y que fomente valores de respeto.

\section{PARTICIPANTES}

En mayo de 2020 se realizó una encuesta dirigida a alumnos de Educación Secundaria (entre 1ำ ESO hasta $2^{\circ}$ Bachillerato). Un total de 182 respondieron a la misma. El $65,4 \%$ de los participantes pertenecieron al género femenino.

\section{METODOLOGÍA Y/O INSTRUMENTOS UTILIZADOS}

Al no hallar encuestas o instrumentos validados que cubriesen los objetivos del estudio, se diseñó un cuestionario ad-hoc inspirado en varias fuentes: "No manipuléis el feminismo: una defensa contra los bulos machistas" (Bernal, 2019) e "Intervención Educativa para la Prevención de la Violencia de Género en los y las Adolescentes” (Alcalde, 2018).

La finalidad del mismo fue la de recoger los mitos y falacias ligados a la relación hombre-mujer, los estereotipos de género y a la liberación de la mujer como parte de la población discriminada para posteriormente, plantearla al alumnado de Educación Secundaria y conocer su perspectiva.

La encuesta quedó conformada por 27 cuestiones, de respuesta tipo Likert con opción múltiple, para mostrar el grado de acuerdo 0 desacuerdo con las afirmaciones propuestas (1, no estoy de acuerdo; 2, algo en desacuerdo; 3, Indiferente; 4, Algo de acuerdo; 5, Estoy de acuerdo). La encuesta se adaptó a la modalidad online y se redactaron las cuestiones utilizando la herramienta Google Forms.

Se obtuvo el permiso del Comité Ético de Investigación del departamento de Enfermería, Fisioterapia y Medicina en la Universidad de Almería, para llevar a cabo esta investigación. Y se solicitó el consentimiento informado de los padres para los menores de 14 años; garantizándoles en todo momento el anonimato y la confidencialidad de los datos.

\section{RESULTADOS ALCANZADOS}

\section{Roles y estereotipos}

Esta sección guarda relación con una selección de estereotipos comunes que caracterizan a ambos géneros y que deriva en la asimilación de un determinado rol en la sociedad.

Se comienza proponiendo una cuestión que plantea las funciones domésticas como una tarea que se encuentra asignada al género femenino. Se obtiene que un $80 \%$ de participantes se muestran en desacuerdo con esta idea, lo que supone una significativa mayoría. Sin embargo, el 15\% restante refleja no estar de acuerdo o mostrarse indiferente con respecto al tema.

Por otro lado, se propone uno de los tópicos más asumidos socialmente referente a las emociones y sentimientos de los hombres y mujeres: "Los hombres lloran menos que las mujeres porque son más fuertes y menos susceptibles a las emociones". El 73,1\% de encuestados se posiciona en "No estoy de acuerdo".

Asimismo, se plantea una cuestión referente a la connotación negativa que puede llegar a tener la forma de vestir de una mujer, en la que un $70 \%$ de las personas encuestadas se posiciona en contra de esta afirmación. El 30\% restante decide no posicionarse u opinar lo contrario.

En cuanto a las relaciones afectivas que se establecen entre parejas heterosexuales, comúnmente se piensa que es el hombre quien debe dar el primer paso. Casi el $30 \%$ de los participantes 


\section{PERCEPCIÓN SOBRE LA IGUALDAD EN JÓVENES ADOLESCENTES Y EL ROL DE LA EDUCACIÓN}

deciden no posicionarse o mostrarse de acuerdo con que "Lo normal es que los hombres den el primer paso cuando les gusta alguien".

Por otro lado, frente a la afirmación "Las mujeres suelen ser más cariñosas que los hombres", se refleja que más de un $45 \%$ de los participantes se posicionan rotundamente en contra de la misma, aunque no existe unanimidad.

\section{El amor}

El amor, representado como el acto más social y humano que caracteriza la especie humana, supone uno de los aspectos más importantes a resaltar, sobre todo entre personas jóvenes, dado que lo comienzan a experimentar de manera significativa a lo largo de toda la etapa de Educación Secundaria.

Se tiende a pensar que los celos representan una manifestación de amor. Sin embargo, la realidad se aleja de esta idea. Ensalzan la desconfianza e inseguridad de una persona sobre sí misma y hacia su pareja. Se plantea la siguiente afirmación: "Si mi pareja se pone celosa de otra persona, me está demostrando que su amor es real". Casi el $80 \%$ de los participantes se posicionan en contra de la afirmación, frente a un $20 \%$ que se muestra indiferente 0 en desacuerdo.

Asimismo, con respecto a las relaciones de pareja, se plantea una cuestión referente al papel del matrimonio como máxima manifestación del amor real. Frente a esta cuestión se encuentra que más del $70 \%$ de los participantes se posiciona en desacuerdo. Sin embargo, casi el 30\% no muestra una opinión sólida o se sitúa favorablemente en relación a esta afirmación.

Por otro lado, se plantea a los encuestados la posibilidad de cambiar algún rasgo del carácter por una pareja. Existe diversidad de opiniones con respecto a este tema a pesar de que destaca una mayoría que se posiciona en contra de la afirmación.

Otra cuestión trata la dependencia en pareja: "Necesito contar con alguien a mi lado para sentirme completo/a". El 70\% se posiciona en contra y un 30\% se muestran "Indiferentes".

Por otro lado, y con relación a las redes sociales, se plantea: "Me parece un acto de confianza compartir la contraseña de Instagram con mi pareja". Un 22\% de los participantes no se ha mostrado en total desacuerdo.

La siguiente cuestión trata sobre los secretos e intimidad en una pareja, donde se propone la idea de compartirlo todo.

Figura 1. "En mi pareja no puede haber secretos, hay que compartirlo todo"

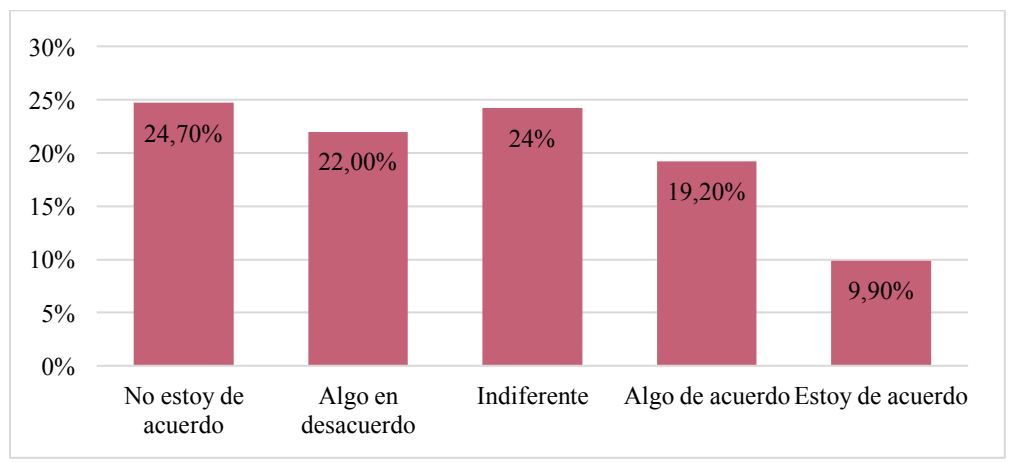


Existe diversidad de opiniones con respecto a la idea. Más del $45 \%$ opina que no hay que compartirlo todo en pareja frente a un $30 \%$ que considera que sí es necesario.

Por último, la cuestión que se plantea es: "Si el/la chico/a que me gusta me dice que no le gusto, me siento frustrado/a y vuelvo a intentarlo". Casi el 10\% está de acuerdo. El 70\% se posiciona en contra de sentirse mal y volver a insistirle a una persona que previamente le ha dado una negativa.

\section{Trabajo}

La siguiente sección guarda relación con el papel de la mujer en el ámbito laboral con respecto a los hombres. Se plantea una cuestión de opción múltiple que trata un concepto cada vez más difundido en medios de comunicación y sobre todo, política: brecha salarial.

Figura 2. Definición de brecha salarial

\begin{tabular}{|l|l|} 
& \\
& La diferencia que hay entre \\
& mujeres y hombres en su \\
& salario a pesar de trabajar lo \\
& mismo y en el mismo cargo \\
& Se refiere a que los hombres \\
& tienen mayores sueldos \\
porque su trabajo y & \\
& rendimiento es mayor \\
& Es un término creado \\
& recientemente para \\
& intentar que las mujeres \\
& cobren más \\
&
\end{tabular}

Casi el $90 \%$ de los participantes tiene claro que la brecha salarial es un término creado para designar la diferencia salarial que existe entre hombres y mujeres a pesar de desempeñar la misma función y la misma jornada laboral.

Ante la afirmación: "No existe diferencia en los sueldos de una mujer y un hombre si desempeñan el mismo cargo", el $40 \%$ de los participantes afirma que esta desigualdad salarial no existe. El $50 \%$ de los participantes está de acuerdo en que es una realidad actual que afecta a la carrera profesional de muchas mujeres.

Respecto a la pregunta: "Existen profesiones que son más acordes a hombres y otras que tienen más relación con las mujeres". Casi el 64\% de los encuestados se muestra en desacuerdo con esta afirmación, pero a pesar de ser mayoría, se debe mostrar que casi el $20 \%$ aún considera que las profesiones tienen relación con el género.

\section{Violencia hacia la mujer}

Esta sección desarrolla el ámbito más devastador y extremo de la manifestación discriminatoria hacia la mujer, pues en el peor de los casos (y como se ha visto, no por ello menos frecuente) el final es la muerte. Se trata sobre todo, la violencia física y la percepción general sobre el tema. Dado que la encuesta también incluía a menores de 16 años, se ha tratado de abordar el tema de la forma más natural y a la vez cautelosa posible.

La primera de las cuestiones hacía referencia al grado de acuerdo que se muestra con respecto a la siguiente afirmación: "Cuando veo en televisión un caso de agresión sexual, siempre pienso que 


\section{PERCEPCIÓN SOBRE LA IGUALDAD EN JÓVENES ADOLESCENTES Y EL ROL DE LA EDUCACIÓN}

si la chica hubiese sido más prudente podría haberlo evitado". Casi el 90\% de los participantes afirma estar en desacuerdo con esta afirmación. Tan sólo un 7,4\% de los encuestados afirma que la causa de este tipo de agresión es evitable por parte de la chica.

Por otro lado, se plantea una cuestión referente a si los hombres que ejercen este tipo de violencia injustificada se debe, en parte, a problemas de salud mental. Casi la mitad de los participantes no están de acuerdo con que la salud mental de un hombre tenga directamente relación con el agredir a una mujer frente a más del 35\% que considera que sí que existe un vínculo entre ambos factores.

Se trata la frecuencia con la que se dan este tipo de situaciones violentas. Casi el $85 \%$ de los participantes se muestran en desacuerdo. Ni siquiera llegan al 10\% los participantes que sí están de acuerdo.

Además, también se hace referencia a los motivos que existen y favorecen este tipo de violencia, por lo que se plantea la siguiente afirmación: "Si una mujer aguanta una relación donde existe violencia durante años, es porque quiere". Casi el $80 \%$ de los encuestados asegura que esta afirmación no es acertada aunque más del 14\% asegura que las mujeres aguantan una relación donde existe violencia durante un determinado tiempo por su propia voluntad.

Se propone la siguiente afirmación: "Siempre ha existido violencia hacia la mujer y es algo inevitable". El $62 \%$ de participantes se posiciona en desacuerdo, y un $25 \%$ que no parece tenerlo tan claro. Sin duda estos resultados son una muestra clara de la falta de interés que posee una parte considerable de personas jóvenes con respecto a erradicar este tipo de situaciones, ya sea porque desvaloran el problema o porque no son conscientes de su gravedad. Si bien se espera algún cambio por parte de la población, creer que es posible, es el primer paso para conseguirlo.

\section{Feminismo}

Al resultado de esta pregunta ¿qué entiendes por feminismo? un $10 \%$ ha señalado la opción que no era la correcta. A pesar de ser una cifra muy reducida, muestra que existe aún una concepción falsa y errónea sobre lo que manifiesta el concepto.

La siguiente afirmación que se propone hace referencia a la historia de la lucha por la igualdad entre hombres y mujeres. Señala que la reivindicación actual que existe por parte del movimiento feminista es totalmente innecesaria, ya que la igualdad se logró hace años. Un ejemplo de ello podría ser el derecho a voto o la emancipación de la mujer. Con respecto a esta idea, más del 10\% opina que los ejemplos citados anteriormente (entre otros) resultan suficientes para que la mujer obtenga los mismos derechos y libertades que los hombres. Frente a estas respuestas, más del $80 \%$ se muestra desfavorable a ello, pues considera que aún es necesario que se produzcan una serie de cambios sociales para conseguir una verdadera equidad entre ambos géneros.

Como punto final a esta sección meramente conceptual, se plantea que los participantes señalen con cuál de las dos oraciones que se proponen se identifican más. Por un lado, con casi un $30 \%$ los encuestados aseguran que "No me considero feminista, ya que creo entre la igualdad entre hombres y mujeres, pero no que exista superioridad por parte de las mujeres". Por otro lado, el $70 \%$ asegura que "Me considero feminista, creo que las mujeres deben conseguir igualdad con respecto a los hombres en todos los ámbitos".

\section{Centros educativos e igualdad}

Como se ha tratado a lo largo del presente trabajo, los centros educativos suponen la herramienta más poderosa junto a las familias para lograr educar a personas con unos valores positivos y tolerantes. En esta sección se trata si los centros realmente llevan a cabo actividades que ayuden y pongan de manifiesto la realidad ante el alumnado. Entre la diversidad de temas de carácter social que necesitan ser mostrados, en este caso, destacamos el de la desigualdad entre géneros. 
Tomando como referencia este punto, se presenta una cuestión de carácter fundamental y a modo general para analizar en qué grado se desarrollan actividades en los centros que fomenten la tolerancia y el respeto entre iguales. Existen diversas maneras de tratar este tema, ya sea de forma transversal en las asignaturas, en forma de charlas o incluso en la propia actitud de los docentes. Sin embargo, cuando se plantea la cuestión "¿Crees que se trata este tema en tu centro?", las respuestas son totalmente opuestas. Es decir, prácticamente la mitad de participantes asegura que sí se trata el tema, frente al "no" de la otra mitad de participantes.

\section{DISCUSIÓN}

Sin lugar a duda, la educación representa el espacio más libre e igualitario para que todo el alumnado pueda recibir una formación de calidad sea cual sea su situación personal o familiar. Representa un contexto social inmediato donde el proceso de aprendizaje puede paliar los estereotipos de género (González, 1999). De esta forma, la educación debe impactar de forma positiva en el alumnado tratando la igualdad con la finalidad de paliar la discriminación, y creando un sistema más participativo, integral y sobre todo, cooperativo.

La revisión de la literatura y los resultados de este cuestionario indican que la desigualdad entre hombres y mujeres se debe a factores relacionados con la biología, pues los roles se siguen en función del sexo de cada persona, que a su vez provocan las diferencias entre ambos (Broverman, Raymond, Broverman, Clarkson y Rosekranzt, 1972).

Además, se subraya la opresión ejercida hacia el genero femenino, victima del concepto de mujer como una persona "debil, sumisa, carinosa y apta para las tareas de cuidado". Este concepto se sustenta en un sistema que organiza la subordinacion de las mujeres y la desigualdad de las mismas en relacion a los varones, por el simple hecho de ser mujeres y las instituciones basicas de su opresion, entre ellas la familia, la maternidad la heterosexualidad obligatoria". (Belloti y Fontenla, 2019, p.207)

Las nuevas generaciones, unidas de forma directa a la educación, suponen el principio del fin. De ahí la importancia a poner de manifiesto su percepción frente a este tema en diversos ámbitos. En lo referente a los estereotipos ligados al genero, se destaca, a nivel general un concepto positivo sobre los mismos. Sin embargo, no sucede lo mismo al tratar el tema del amor, pues los porcentajes varian a favor de la toxicidad y perdida de la privacidad personal. Lo mismo sucede con respecto al trabajo, donde la mitad de los participantes no consideran que exista discriminacion en este ambito.

Por otro lado, convenientemente diferenciada del resto de secciones por su delicada situacion, se habla de la violencia de genero. Sin duda, el apartado con mas variedad de opinion, lo que lo posiciona como el aspecto sobre el que existe mayor desconocimiento a nivel general. No existe unanimidad frente a cuestiones que no deberian ser motivo de "debate", como lo son las victimas por violencia machista. Ello reitera la importancia de la educacion y su involucracion con el mismo.

\section{CONCLUSIONES}

A modo de conclusion general sobre los datos obtenidos, se senala la educacion como principal facilitadora de informacion al alumnado con respecto a la realidad sufrida por mujeres. Es necesario que el alumnado conozca que deben ser libres y pueden alejarse de aquello que la sociedad le ha asignado al nacer; que todas las profesiones son aptas para todas las personas y que el salario no debe girar en funcion a la biologia de las mismas; que una relacion de amor sana esta caracterizada por la confianza y el consentimiento, pero nunca por la intromision del otro; que la violencia hacia la mujer no es fruto de problemas psiquicos de algunos hombres, sino que existe de forma sistematica; y por ultimo, que "feminismo" es el nombre que recibe el abogar por la equidad de las personas y el papel que merecen las mujeres en la sociedad. 


\section{PERCEPCIÓN SOBRE LA IGUALDAD EN JÓVENES ADOLESCENTES Y EL ROL DE LA EDUCACIÓN}

\section{REFERENCIAS BIBLIOGRÁFICAS}

Alcalde, D. (2018). Intervención educativa para la prevención de la violencia de género en los y las adolescentes del centro educativo IES Tartessos. Universidad de Sevilla. Recuperado el 13 de mayo de: https://idus.us.es/bitstream/handle/11441/85568/GRAD0\%20EN\%20PEDAGOGÍA\% 20IMPRIMIR\%20TFG.pdf?sequence=1\&isAllowed=y

Belloti, M y Fontenla M. (2008). Patriarcado. En Gamba, S. (coord.). Se va a caer: Conceptos básicos de los feminismos (pp. 207-212). La Plata: Pixel

Bernal, A. (2019). No manipuleis el feminismo : una defensa contra los bulos machistas. Espassa. Recuperado el 20 de abril de: https://nubereader.odilotk.es/\#/161ff6ce-337140ebb565eeef29840294/5568530dccac9fbe0d77e8fa72019515295d3c951b3c39cc80076993d 99de8ae

Broverman, I., Raymond, S., Broverman, D., Clarkson, F. y Rosekranzt, P. (1972). Sex-role stereotypes: a current appraisal. Journal of Social Issues. No. 28, 59- 79.

Colás, P. y Villaciervos P. (2007). La interiorización de los estereotipos de género en jóvenes y adoIescentes. Revista De Investigación Educativa, 25(1), 35-38. Recuperado el 20 de abril de https://revistas.um.es/rie/article/view/96421

Extremera, A. B., y Montero, P. J. R. (2009). Tratamiento educativo de la coeducacion y la igualdad de sexos en el contexto escolar y en espacial en Educacion Fisica. Aula abierta, 37(2), 111- 122.

González, B. (1999). Los estereotipos como factor de socialización en el género. Comunicar, n. 12. Huelva. Recuperado el 2 de mayo de: https://www.redalyc.org/pdf/158/15801212.pdf

Martori, M. S. (2009). La escuela mixta ¿garantia de coeducacion? Participacion educativa, (11), 9497.

Raedo, G. (2014). Igualdad de género, importancia de la coeducación en la actualidad. Universidad de Valladolid. Recuperado el 15 de mayo de: https://uvadoc.uva.es/handle/10324/6674 